Commun. Fac. Sci. Univ. Ank. Ser. A1 Math. Stat.

Volume 69, Number 1, Pages 441449(2020)

DOI: $10.31801 /$ cfsuasmas.585800

ISSN 1303-5991 E-ISSN 2618-6470

http://communications.science.ankara.edu.tr/index.php?series=A1

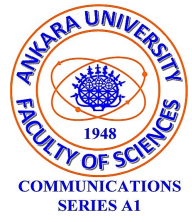

\title{
GREEN FUNCTION AND RESOLVENT OPERATOR OF A SCHRÖDINGER EQUATION WITH GENERAL POINT INTERACTION
}

\author{
EMEL YILDIRIM
}

\begin{abstract}
In this paper, we investigate the time independent Schrödinger equation which has complex valued potential function under the general point interaction. We construct Green function of this problem and we find the resolvent of the problem in terms of Green function.
\end{abstract}

\section{INTRODUCTION}

In mathematics, a Green's function is used to solve inhomogeneous differential equations under various initial conditions or boundary conditions. The term is also used in physics, quantum mechanics, engineering and quantum field theory to signify various types of correlation functions. It has been shown that should be taken into consideration a perturbation series to an infinite in order to obtain a deep insight not attainable by a finite-order treatment thanks to Green's functions in many quantum-mechanical applications. Therefore, these functions are very important in many disciplines and much work has been done on this theory. Especially, Green's functions have been used in investigating the completeness property of the set of eigenfunctions of self-adjoint operators and in proving the expansion theorem for an arbitrary function in terms of the complete set obtained from a Sturm-Liouville operator [9]. Also, it has been obtained by Sakurai that one of the easiest ways to identify the peculiar feature of a particular quantum mechanics, which contrasts with classical mechanics, is to convert the action integral for a classical motion into the form involving the Green's functions of the Schrödinger equation [15] . As a result of these studies, many researchers have examined of Green's functions of Schrödinger equation with some initial conditions or boundary conditions [20, 16].

Received by the editors: July 02, 2019; Accepted: October 15, 2019.

2010 Mathematics Subject Classification. Primary 34B27, 34L05; Secondary 81Q10.

Key words and phrases. Green function, resolvent operator, Schrödinger operators, point interaction.

(C)2020 Ankara University Communications Faculty of Sciences University of Ankara-Series A1 Mathematics and Statistics 
The Green's functions of the Schrödinger equation for the simplest quantum mechanical systems have been investigated in [7]. Sturm Liouville operator with eigenparameter dependent boundary conditions and transmission conditions at a finite number of interior points have been studied and Green's function has been obtained for this problem in [12.

Additionally, differential equations with transmission condition has an important role for many branches of sciences. In particularly, they are used in quantum mechanics and atomic physics. Because some of them are considered to represent many physical systems quantitatively. It is known that there are four parameters point interactions that can be represent as self-adjoint extensions of the nonrelativistic kinetic energy operator in one-dimensional quantum mechanics [10, 11. For the mathematical theory of differential equations with point interaction, we refer to the monographs 4, 5. In the literature, many various type of differential equations have been investigated under the point interaction [1, 2, 3, 8, 14, 19. In particular, dissipative boundary value problems with point interaction have been studied in [6, 17, 18.

In this paper, our aim is to find the Resolvent operator of the Schrödinger equation under general point interaction by constructing the Green function.

\section{Some Properties of Solutions of the Schrödinger Equation on}

\section{Whole Axis}

Let us consider the time independent Schrödinger equation

$$
-\psi^{\prime \prime}(x)+q(x) \psi(x)=\lambda^{2} \psi(x), \quad x \in \mathbb{R} \backslash\{0\}
$$

where $\lambda$ is spectral parameter, $q(x)$ is complex valued function. The equation (1) has bounded solutions $e_{ \pm}(x, \lambda)$ which they satisfy following limit conditions.

$$
\lim _{x \rightarrow \pm \infty} \psi(x) e^{\mp i \lambda x}=1, \quad \lambda \in \overline{\mathbb{C}}_{+}=\{\lambda: \lambda \in \mathbb{C}, \operatorname{Im} \lambda \geq 0\} .
$$

The solutions $e_{ \pm}(x, \lambda)$ are called Jost solution of equation (1) and they have the following representations

$$
\begin{aligned}
& e_{-}(x, \lambda)=e^{-i \lambda x}+\int_{-\infty}^{x} K^{-}(x, t) e^{-i \lambda t} d t, \lambda \in \overline{\mathbb{C}}_{+}, \quad-\infty<x<0 \\
& e_{+}(x, \lambda)=e^{i \lambda x}+\int_{x}^{\infty} K^{+}(x, t) e^{i \lambda t} d t, \quad \lambda \in \overline{\mathbb{C}}_{+}, 0<x<\infty
\end{aligned}
$$

under the condition

$$
\int_{-\infty}^{\infty}(1+|x|)|q(x)| d x<\infty .
$$


$K^{+}(x, t)$ and $K^{-}(x, t)$ are called Kernel functions and they are defined as follows.

$$
\begin{aligned}
K^{+}(x, t)= & \frac{1}{2} \int_{\frac{x+t}{2}}^{\infty} q(s) d s+\frac{1}{2} \int_{x}^{\frac{x+t}{2}} \int_{x+t-s}^{t-x+s} q(s) K^{+}(s, r) d s d r \\
& +\frac{1}{2} \int_{\frac{x+t}{2}}^{\infty} \int_{s}^{t-x+s} q(s) K^{+}(s, r) d s d r \\
K^{-}(x, t)= & \frac{1}{2} \int_{-\infty}^{\frac{x+t}{2}} q(s) d s+\frac{1}{2} \int_{x}^{\frac{x+t}{2}} \int_{x+t-s}^{t-x+s} q(s) K^{-}(s, r) d s d r \\
& +\frac{1}{2} \int_{-\infty}^{\frac{x+t}{2}} \int_{s}^{t-x+s} q(s) K^{-}(s, r) d s d r
\end{aligned}
$$

Moreover, these functions are continuously differentiable with respect to their arguments and satisfy the following inequalities:

$$
\begin{aligned}
\left|K^{ \pm}(x, t)\right| & \leq c \sigma^{ \pm}\left(\frac{x+t}{2}\right) \\
\left|K_{x}^{ \pm}(x, t) \pm \frac{1}{4}\right| q\left(\frac{x+t}{2}\right)|| & \leq c \sigma^{ \pm}\left(\frac{x+t}{2}\right) \\
\left|K_{t}^{ \pm}(x, t) \pm \frac{1}{4}\right| q\left(\frac{x+t}{2}\right)|| & \leq c \sigma^{ \pm}\left(\frac{x+t}{2}\right)
\end{aligned}
$$

where

$$
\sigma^{+}(t)=\int_{x}^{\infty}|q(t)| d t, \quad \sigma^{-}(t)=\int_{-\infty}^{x}|q(t)| d t
$$

and $c>0$ is a constant. Furthermore, $\widehat{e}_{ \pm}(x, \lambda)$ are unbounded solutions of equation (1) which they satisfy

$$
\lim _{x \rightarrow \pm \infty} \psi(x) e^{ \pm i \lambda x}=1
$$

13. 


\section{Green Function of the Schrödinger Equation with General Point} INTERACTION

In this section, we shall construct Green function of the Schrödinger equation (1) with the general point interaction

$$
\left(\begin{array}{l}
\psi_{+}(0, \lambda) \\
\psi_{+}^{\prime}(0, \lambda)
\end{array}\right)=\left(\begin{array}{ll}
a & b \\
c & d
\end{array}\right)\left(\begin{array}{l}
\psi_{-}(0, \lambda) \\
\psi_{-}^{\prime}(0, \lambda)
\end{array}\right)
$$

where $a, b, c, d \in \mathbb{R}$ and $a d-b c \neq 0$ and we shall find the Resolvent operator of this problem. Green function of the problem (1)-(8) is a solution to following non-homogeneous differential equation

$$
-\psi^{\prime \prime}(x)+q(x) \psi(x)-\lambda^{2} \psi(x)=f(x), \quad x \in \mathbb{R} \backslash\{0\}
$$

To obtain the general solution of this equation, we need to find solutions of equation (1). The two linear independent solutions of its can be given as:

$$
\begin{aligned}
& U(x, \lambda)= \begin{cases}U_{+}(x, \lambda), & x>0 \\
U_{-}(x, \lambda), & x<0\end{cases} \\
& =\left\{\begin{array}{c}
e_{+}(x, \lambda), \quad x>0 \\
\alpha(\lambda) e_{-}(x, \lambda)+\beta(\lambda) \widehat{e}_{-}(x, \lambda), \quad x<0
\end{array}, \quad \lambda \in \mathbb{C}\right. \\
& V(x, \lambda)= \begin{cases}V_{+}(x, \lambda), & x>0 \\
V_{-}(x, \lambda), & x<0\end{cases} \\
& =\left\{\begin{array}{c}
\hat{\alpha}(\lambda) e_{+}(x, \lambda)+\hat{\beta}(\lambda) \widehat{e}_{+}(x, \lambda), \quad x<0 \\
e_{-}(x, \lambda), \quad x<0
\end{array}, \quad \lambda \in \mathbb{C}\right.
\end{aligned}
$$

where $\widehat{e}_{ \pm}(x, \lambda)$ are unbounded solutions and $e_{ \pm}(x, \lambda)$ are bounded solutions of equation (1). By using the solutions $U(x, \lambda)$ and $V(x, \lambda)$ and the condition (8), we get

$$
\begin{aligned}
e_{+}(0, \lambda)= & a \alpha(\lambda) e_{-}(0, \lambda)+a \beta(\lambda) \widehat{e}_{-}(0, \lambda) \\
& +b \alpha(\lambda) e_{-}^{\prime}(0, \lambda)+b \beta(\lambda) \widehat{e}_{-}^{\prime}(0, \lambda) \\
& \\
e_{+}^{\prime}(0, \lambda)= & c \alpha(\lambda) e_{-}(0, \lambda)+c \beta(\lambda) \widehat{e}_{-}(0, \lambda) \\
& +d \alpha(\lambda) e_{-}^{\prime}(0, \lambda)+d \beta(\lambda) \widehat{e}_{-}^{\prime}(0, \lambda) \\
\widehat{\alpha}(\lambda) e_{+}(0, \lambda)+\widehat{\beta}(\lambda) \widehat{e}_{+}(0, \lambda)= & a e_{-}(0, \lambda)+b e_{-}^{\prime}(0, \lambda) \\
\widehat{\alpha}(\lambda) e_{+}^{\prime}(0, \lambda)+\widehat{\beta}(\lambda) \widehat{e}_{+}(0, \lambda)= & c e_{-}(0, \lambda)+d e_{-}^{\prime}(0, \lambda) .
\end{aligned}
$$


For these equations to be true, $\alpha(\lambda), \beta(\lambda), \widehat{\alpha}(\lambda)$ and $\widehat{\beta}(\lambda)$ must be as follows, respectively.

$$
\begin{gathered}
\alpha(\lambda)=\frac{1}{2 i \lambda(a d-b c)}\left(\begin{array}{c}
c \widehat{e}_{-}(0, \lambda) e_{+}(0, \lambda)+d e_{+}(0, \lambda) \widehat{e}_{-}^{\prime}(0, \lambda) \\
-a \widehat{e}_{-}(0, \lambda) e_{+}^{\prime}(0, \lambda)-b e_{+}^{\prime}(0, \lambda) \widehat{e}_{-}^{\prime}(0, \lambda)
\end{array}\right) \\
\beta(\lambda)=\frac{1}{2 i \lambda(a d-b c)}\left(\begin{array}{c}
a e_{-}(0, \lambda) e_{+}^{\prime}(0, \lambda)+b e_{-}^{\prime}(0, \lambda) e_{+}^{\prime}(0, \lambda) \\
-c e_{-}(0, \lambda)-d e_{-}^{\prime}(0, \lambda) e_{+}(0, \lambda)
\end{array}\right) \\
\widehat{\alpha}(\lambda)=\frac{1}{2 i \lambda}\left(\begin{array}{c}
a e_{-}(0, \lambda) \widehat{e}_{+}^{\prime}(0, \lambda)+b e_{-}^{\prime}(0, \lambda) \widehat{e}_{+}^{\prime}(0, \lambda) \\
-c e_{-}(0, \lambda) \widehat{e}_{+}(0, \lambda)-d e_{-}^{\prime}(0, \lambda) \widehat{e} \widehat{e}_{+}(0, \lambda)
\end{array}\right) \\
\widehat{\beta}(\lambda)=\frac{1}{2 i \lambda}\left(\begin{array}{c}
c e_{-}(0, \lambda) e_{+}^{\prime}(0, \lambda)-d e_{-}^{\prime}(0, \lambda) e_{+}(0, \lambda) \\
-a e_{-}(0, \lambda) e_{+}^{\prime}(0, \lambda)+b e_{-}^{\prime}(0, \lambda) e_{+}^{\prime}(0, \lambda)
\end{array}\right)
\end{gathered}
$$

From these equations, it is seen that $\widehat{\beta}(\lambda)$ can be written in terms of $\beta(\lambda)$ such that

$$
\widehat{\beta}(\lambda)=(a d-b c) \beta(\lambda) .
$$

Moreover, since the linear combination of solutions of a differential equation is the solution of this equation, the general solution of equation (1) can be given by using solutions $U(x, \lambda)$ and $V(x, \lambda)$

$$
\begin{aligned}
y(x, \lambda) & = \begin{cases}y_{-}(x, \lambda), & x<0 \\
y_{+}(x, \lambda), & x>0\end{cases} \\
& = \begin{cases}c_{1} U_{-}(x, \lambda)+c_{2} V_{-}(x, \lambda), & x<0 \\
d_{1} U_{+}(x, \lambda)+d_{2} V_{+}(x, \lambda), & x>0\end{cases}
\end{aligned}
$$

where $c_{1}, c_{2}, d_{1}$ and $d_{2}$ are constant. If we use the method of variation of constants, we find the general solution of equation (9) in the form

$$
\begin{aligned}
\widetilde{y}(x, \lambda) & = \begin{cases}\widetilde{y_{-}}(x, \lambda), & x<0 \\
\widetilde{y_{+}}(x, \lambda), & x>0\end{cases} \\
& = \begin{cases}c_{1}(x) U_{-}(x, \lambda)+c_{2}(x) V_{-}(x, \lambda), & x<0 \\
d_{1}(x) U_{+}(x, \lambda)+d_{2}(x) V_{+}(x, \lambda), & x>0\end{cases}
\end{aligned}
$$

where $c_{1}(x), c_{2}(x)$ and $d_{1}(x), d_{2}(x)$ satisfy the following equation systems

$$
\begin{aligned}
& c_{1}^{\prime}(x) U_{-}(x, \lambda)+c_{2}^{\prime}(x) V_{-}(x, \lambda)=0 \\
& c_{1}^{\prime}(x) U_{-}^{\prime}(x, \lambda)+c_{2}^{\prime}(x) V_{-}^{\prime}(x, \lambda)=-f(x)
\end{aligned}
$$


and

$$
\begin{aligned}
& d_{1}^{\prime}(x) U_{+}(x, \lambda)+d_{2}^{\prime}(x) V_{+}(x, \lambda)=0 \\
& d_{1}^{\prime}(x) U_{+}^{\prime}(x, \lambda)+d_{2}^{\prime}(x) V_{+}^{\prime}(x, \lambda)=-f(x) .
\end{aligned}
$$

When the Cramer Method's is applied to these equations, the following equations are obtained.

$$
\begin{aligned}
c_{1}^{\prime}(x) & =\frac{V_{-}(x, \lambda) f(x)}{W\left[U_{-}(x, \lambda), V_{-}(x, \lambda)\right]} \\
c_{2}^{\prime}(x) & =\frac{-U_{-}(x, \lambda) f(x)}{W\left[U_{-}(x, \lambda), V_{-}(x, \lambda)\right]} \\
d_{1}^{\prime}(x) & =\frac{V_{+}(x, \lambda) f(x)}{W\left[U_{+}(x, \lambda), V_{+}(x, \lambda)\right]} \\
d_{2}^{\prime}(x) & =\frac{-U_{+}(x, \lambda) f(x)}{W\left[U_{+}(x, \lambda), V_{+}(x, \lambda)\right]}
\end{aligned}
$$

Considering that $U_{+}(x, \lambda)$ and $V_{+}(x, \lambda)$ are defined on $(0, \infty)$ and $U_{-}(x, \lambda)$ and $V_{-}(x, \lambda)$ are defined on $(-\infty, 0), c_{1}(x), c_{2}(x), d_{1}(x)$ and $d_{2}(x)$ can be expressed by the following integral equations;

$$
\begin{aligned}
& c_{1}(x)=\alpha_{1}+\int_{-\infty}^{x} \frac{V_{-}(t, \lambda) f(t)}{W\left[U_{-}(t, \lambda), V_{-}(t, \lambda)\right]} d t, \\
& c_{2}(x)=\alpha_{2}+\int_{x}^{0} \frac{-U_{-}(t, \lambda) f(t)}{W\left[U_{-}(t, \lambda), V_{-}(t, \lambda)\right]} d t, \\
& d_{1}(x)=\alpha_{3}+\int_{0}^{x} \frac{V_{+}(t, \lambda) f(t)}{W\left[U_{+}(t, \lambda), V_{+}(t, \lambda)\right]} d t, \\
& d_{2}(x)=\alpha_{4}+\int_{x}^{\infty} \frac{-U_{+}(t, \lambda) f(t)}{W\left[U_{+}(t, \lambda), V_{+}(t, \lambda)\right]} d t .
\end{aligned}
$$

If we consider these equation in (14), the general solution of equation (9) can be given as

$$
\begin{aligned}
\widetilde{y_{-}}(x, \lambda)= & \alpha_{1} U_{-}(x, \lambda)+U_{-}(x, \lambda) \int_{-\infty}^{x} \frac{V_{-}(t, \lambda) f(t)}{W\left[U_{-}(t, \lambda), V_{-}(t, \lambda)\right]} d t \\
& +\alpha_{2} V_{-}(x, \lambda)-V_{-}(x, \lambda) \int_{x}^{0} \frac{U_{-}(t, \lambda) f(t)}{W\left[U_{-}(t, \lambda), V_{-}(t, \lambda)\right]} d t .
\end{aligned}
$$




$$
\begin{aligned}
\widetilde{y_{+}}(x, \lambda)= & \alpha_{3} U_{+}(x, \lambda)+U_{+}(x, \lambda) \int_{0}^{x} \frac{V_{+}(t, \lambda) f(t)}{W\left[U_{+}(t, \lambda), V_{+}(t, \lambda)\right]} d t \\
& +\alpha_{4} V_{+}(x, \lambda)-V_{+}(x, \lambda) \int_{x}^{\infty} \frac{U_{+}(t, \lambda) f(t)}{W\left[U_{+}(t, \lambda), V_{+}(t, \lambda)\right]} d t .
\end{aligned}
$$

Since $\widetilde{y_{-}}(x, \lambda) \in L_{2}(-\infty, 0)$ and $\widetilde{y_{+}}(x, \lambda) \in L_{2}(0, \infty), \alpha_{1}, \alpha_{2}, \alpha_{3}$ and $\alpha_{4}$ must be equal to zero. Therefore the general solution of non-homogeneous equation (9) has the following form:

$$
\widetilde{y}(x, \lambda)=\left\{\begin{array}{cc}
\int_{-\infty}^{x} \frac{V_{-}(t, \lambda) U_{-}(x, \lambda) f(t)}{W\left[U_{-}(t, \lambda), V_{-}(t, \lambda)\right]} d t+\int_{x}^{0} \frac{-U_{-}(t, \lambda) V_{-}(x, \lambda) f(t)}{W\left[U_{-}(t, \lambda), V_{-}(t, \lambda)\right]} d t, \quad x<0 \\
\int_{0}^{x} \frac{V_{+}(t, \lambda) U_{+}(x, \lambda) f(t)}{W\left[U_{+}(t, \lambda), V_{+}(t, \lambda)\right]} d t+\int_{x}^{\infty} \frac{-U_{+}(t, \lambda) V_{+}(x, \lambda) f(t)}{W\left[U_{+}(t, \lambda), V_{+}(t, \lambda)\right]} d t, \quad x>0
\end{array}\right.
$$

Moreover, by using the representations of $U(x, \lambda)$ and $V(t, \lambda)$, this formula can be rewritten in the form

$$
\widetilde{y}(x, \lambda)=U(x, \lambda) \int_{-\infty}^{x} \frac{V(t, \lambda) f(t)}{W[U(t, \lambda), V(t, \lambda)]} d t-V(x, \lambda) \int_{x}^{\infty} \frac{U(t, \lambda) f(t)}{W[U(t, \lambda), V(t, \lambda)]} d t
$$

Consequently, we can write the Green function of the problem (1)-(8).

$$
G(x, t ; \lambda)=\left\{\begin{array}{cc}
\frac{U(x, \lambda) V(t, \lambda)}{W[U(x, \lambda), V(x, \lambda)]}, & -\infty<x \leq t, x \neq t \neq 0 \\
\frac{V(x, \lambda) U(t, \lambda)}{W[U(x, \lambda), V(x, \lambda)]}, & x \leq t<\infty, x \neq t \neq 0
\end{array}\right.
$$

The Wronksian of the solutions $U(x, \lambda)$ and $V(x, \lambda)$ can be calculated easily by using the definiton of them. For $\mathrm{x}>0$,

$$
\begin{aligned}
W[U(x, \lambda), V(x, \lambda)]= & \left|\begin{array}{cc}
e_{+}(x, \lambda) & \widehat{\alpha}(\lambda) e_{+}(x, \lambda)+\widehat{\beta}(\lambda) \widehat{e}_{+}(x, \lambda) \\
e_{+}^{\prime}(x, \lambda) & \widehat{\alpha}(\lambda) e_{+}^{\prime}(x, \lambda)+\widehat{\beta}(\lambda) \widehat{e}_{+}^{\prime}(x, \lambda)
\end{array}\right| \\
= & \widehat{\alpha}(\lambda) e_{+}^{\prime}(x, \lambda) e_{+}(x, \lambda)+\widehat{\beta}(\lambda) \widehat{e}_{+}^{\prime}(x, \lambda) e_{+}(x, \lambda) \\
& -\widehat{\alpha}(\lambda) e_{+}(x, \lambda) e_{+}^{\prime}(x, \lambda)-\widehat{\beta}(\lambda) \widehat{e}_{+}(x, \lambda) e_{+}^{\prime}(x, \lambda) \\
= & \widehat{\beta}(\lambda)\left(e_{+}(x, \lambda) \widehat{e}_{+}^{\prime}(x, \lambda)-\widehat{e}_{+}(x, \lambda) e_{+}^{\prime}(x, \lambda)\right) \\
= & -2 i \lambda \widehat{\beta}(\lambda)
\end{aligned}
$$


and for $\mathrm{x}<0$,

$$
\begin{aligned}
W[U(x, \lambda), V(x, \lambda)]= & \left|\begin{array}{cc}
\alpha(\lambda) e_{-}(x, \lambda)+\beta(\lambda) \widehat{e}_{-}(x, \lambda), & e_{-}(x, \lambda) \\
\alpha(\lambda) e_{-}^{\prime}(x, \lambda)+\beta(\lambda) \widehat{e}_{-}^{\prime}(x, \lambda), & e_{-}^{\prime}(x, \lambda)
\end{array}\right| \\
= & \alpha(\lambda) e_{-}(x, \lambda) e_{-}^{\prime}(x, \lambda)+\beta(\lambda) \widehat{e}_{-}(x, \lambda) e_{-}^{\prime}(x, \lambda) \\
& -\alpha(\lambda) e_{-}^{\prime}(x, \lambda) e_{-}(x, \lambda)-\beta(\lambda) \widehat{e}_{-}^{\prime}(x, \lambda) e_{-}(x, \lambda) \\
= & -\beta(\lambda)\left(e_{-}(x, \lambda) \widehat{e}_{-}^{\prime}(x, \lambda)-\widehat{e}_{-}(x, \lambda) e_{-}^{\prime}(x, \lambda)\right) \\
= & -2 i \lambda \beta(\lambda) .
\end{aligned}
$$

In view of (12) and (17), the Wronskian can be arranged.

$$
W[U(x, \lambda), V(x, \lambda)]=\left\{\begin{array}{cc}
-2 i \lambda(a d-b c) \beta(\lambda), & x>0 \\
-2 i \lambda \beta(\lambda), & x<0
\end{array}\right.
$$

Consequently, the Resolvent operator of this problem can be given as follows.

$$
R_{\lambda}(f)=\int_{-\infty}^{\infty} G(x, t ; \lambda) f(t) d t .
$$

Acknowledgement. The author emits her sincere thanks to Prof. Elgiz Bairamov for his kind interest, valuable suggestions and comments. Moreover, the author thanks two referees for the valuable suggestion that led to a significant improvement over the previous version of manuscript.

\section{REFERENCES}

[1] Albeverio, S. and Leonid, N., Schrödinger operators with nonlocal point interactions, J. Math. Anal. Appl., 332 (2007), 884-895.

[2] Albeverio, S. and Kurasov, P., Pseudo-differential operators with point interactions, Lett. Math. Phys., 41 (1997). 79-92.

[3] Allahverdiev, B., Bairamov, E. and Ugurlu, E., Eigenparameter dependent Sturm-Liouville problems in boundary conditions with transmission conditions, J. Math. Anal. Appl., 401 (2013), 388-396.

[4] Bainov, D.D. and Simeovov, P.S., Impulsive differential equations: asymptotic properties of the solutions, World Scentific, Singapore, 1995.

[5] Bainov, D.D. and Simeovov, P.S., Oscilation theory of impulsive differential equations, International Publications, Orlando, 1998.

[6] Bairamov, E. and Ugurlu, E., Krein's theorems for a dissipative boundary value transmission problem, Complex Anal. Oper. Theory, 4 (2013), 831-842.

[7] Bakhrakh, V. L. and Vetchinkin, S. I., Green's functions of the Schrödinger equation for the simplest systems, Theor. Math. Phys., 6:3 (1971), 283-290.

[8] Benehohra, M., Henderson, J. and Ntouyas, S., Impulsive differential equations and inclusions, Hindawi Publishing Corporation, New York, 2006.

[9] Courant, R. and Hilbert, D., Methoden der Mathematischen Physik, Springer, Berlin, 1937.

[10] Coutinho, F.A.B., Nogami, Y. and Tomio, L. Many-body system with a four-parameter family of point interactions in one dimension, J. Phys. A. Math. Gen., 32, (1999). 
GREEN FUNCTION AND RESOLVENT OPERATOR OF A SCHRÖDINGER EQUATION449

[11] Coutinho, F.A.B., Nogami, Y. and Perez, J.F., Generalized point interactions in onedimensional quantum mechanics, J. Phys. A. Math. Gen., 30 (1997), 3937-3945.

[12] Dehghani, I. and Akbarfam, A.J., Resolvent operator and self-adjointness of Sturm-Liouville operators with a finite number of transmission conditions, Mediterr. J. Math., 11 (2014), 447.

[13] Marchenko, V.A., Sturm-Liouville operators and applications, Birkhöuser, Basel, 1986.

[14] Nenov, S.I., Impulsive controllability and optimization problems in population dynamics, Nonlinear Anal., 36 (1999), 881-890.

[15] Sakurai, J.J., Modern Quantum Mechanics, MA, Addison-Wesley, 1985.

[16] Tsaur, G. and Wang, J., Constructing Green functions of the Schrödinger equation by elementary transformations, Am. J. Phys. 74 (2006), 600.

[17] Ugurlu, E. and Bairamov, E., Spectral analysis of eigenparameter dependent boundary value transmission problems, J. Math. Anal. Appl., 1 (2014), 482-494.

[18] Ugurlu, E. and Bairamov, E., The spectral analysis of a nuclear resolvent operator associated with a second order dissipative differential operator. Comput. Methods Funct. Theory, 17(2) (2016).

[19] Yildirim, E., Bound states and spectral singularities of an impulsive Schrödinger equation, Turk. J. Math., 42 (2018), 1670-1679.

[20] Zhang, X. and Sun, J., Green function of fourth-order differential operator with eigenparameter-dependent boundary and transmission conditions, Acta Math. Appl. Sin. Engl. Ser., 33 (2017), 311.

Current address: Emel YILDIRIM: Department of Mathematics Ankara University, Faculty of Science 06100 Ankara, Turkey

E-mail address: emelyildirim@ankara.edu.tr

ORCID Address: http://orcid.org/0000-0002-9894-2113 